\title{
Article \\ Structure-Based Design, Docking and Binding Free Energy Calculations of A366 Derivatives as Spindlin1 Inhibitors
}

\author{
Chiara Luise ${ }^{1}$, Dina Robaa ${ }^{1}$, Pierre Regenass ${ }^{2}$, David Maurer ${ }^{2}{ }^{(}$, , Dmytro Ostrovskyi ${ }^{2}$, Ludwig Seifert ${ }^{3}$, \\ Johannes Bacher ${ }^{3}$, Teresa Burgahn ${ }^{3}$, Tobias Wagner ${ }^{3}$, Johannes Seitz ${ }^{3}$, Holger Greschik ${ }^{4}$, Kwang-Su Park ${ }^{5} \mathbb{D}_{\text {, }}$
} Yan Xiong ${ }^{5}$, Jian Jin ${ }^{5}{ }^{-}$, Roland Schüle ${ }^{4}$, Bernhard Breit ${ }^{2}$, Manfred Jung ${ }^{3,6}{ }^{(\mathbb{D}}$ and Wolfgang Sippl ${ }^{1, * \mathbb{C}}$

1 Institute of Pharmacy, Martin Luther University of Halle-Wittenberg, Kurt-Mothes-Str. 3, 06120 Halle (Saale), Germany; luisechi@hotmail.it (C.L.); dina.robaa@pharmazie.uni-halle.de (D.R.)

2 Institute for Organic Chemistry, University of Freiburg, Albertstr. 21, 79104 Freiburg, Germany; pm.regenass@gmail.com (P.R.); DM.Maurer@web.de (D.M.); dmytroostrovskyi@googlemail.com (D.O.); bernhard.breit@chemie.uni-freiburg.de (B.B.)

3 Institute of Pharmaceutical Sciences, University of Freiburg, Albertstr. 25, 79104 Freiburg, Germany; ludwig.seifert@pharmazie.uni-freiburg.de (L.S.); johannes.bacher@pharmazie.uni-freiburg.de (J.B.); teresa.burgahn@web.de (T.B.); dr.wagner.tobias@gmail.com (T.W.);

johannes.seifert@pharmazie.uni-freiburg.de (J.S.); manfred.jung@pharmazie.uni-freiburg.de (M.J.)

4 Department of Urology and Center for Clinical Research, University Freiburg Medical Center, Breisacherstr. 66, 79106 Freiburg, Germany; holger.greschik@uniklinik-freiburg.de (H.G.); roland.schuele@uniklinik-freiburg.de (R.S.)

5 Mount Sinai Center for Therapeutics Discovery, Departments of Pharmacological Sciences and Oncological Sciences, Tisch Cancer Institute, Icahn School of Medicine at Mount Sinai, New York, NY 10029, USA; kwang-su.park@mssm.edu (K.-S.P.); yan.xiong@mssm.edu (Y.X.); jian.jin@mssm.edu (J.J.)

updates

Citation: Luise, C.; Robaa, D.; Regenass, P.; Maurer, D.;

Ostrovskyi, D.; Seifert, L.; Bacher, J.; Burgahn, T.; Wagner, T.; Seitz, J.; et al. Structure-Based Design, Docking and Binding Free Energy Calculations of A366 Derivatives as Spindlin1 Inhibitors. Int. J. Mol. Sci. 2021, 22, 5910. https://doi.org/10.3390/ijms 22115910

Academic Editor: Giulio Poli

Received: 5 May 2021

Accepted: 26 May 2021

Published: 31 May 2021

Publisher's Note: MDPI stays neutral with regard to jurisdictional claims in published maps and institutional affiliations.

Copyright: (C) 2021 by the authors. Licensee MDPI, Basel, Switzerland. This article is an open access article distributed under the terms and conditions of the Creative Commons Attribution (CC BY) license (https:// creativecommons.org/licenses/by/ $4.0 /)$.
6 CIBSS-Centre for Integrative Biological Signalling Studies, University of Freiburg, 79104 Freiburg, Germany

* Correspondence: wolfgang.sippl@pharmazie.uni-halle.de

Abstract: The chromatin reader protein Spindlin1 plays an important role in epigenetic regulation, through which it has been linked to several types of malignant tumors. In the current work, we report on the development of novel analogs of the previously published lead inhibitor A366. In an effort to improve the activity and explore the structure-activity relationship (SAR), a series of 21 derivatives was synthesized, tested in vitro, and investigated by means of molecular modeling tools. Docking studies and molecular dynamics (MD) simulations were performed to analyze and rationalize the structural differences responsible for the Spindlin1 activity. The analysis of MD simulations shed light on the important interactions. Our study highlighted the main structural features that are required for Spindlin1 inhibitory activity, which include a positively charged pyrrolidine moiety embedded into the aromatic cage connected via a propyloxy linker to the 2-aminoindole core. Of the latter, the amidine group anchor the compounds into the pocket through salt bridge interactions with Asp184. Different protocols were tested to identify a fast in silico method that could help to discriminate between active and inactive compounds within the A366 series. Rescoring the docking poses with MM-GBSA calculations was successful in this regard. Because A366 is known to be a G9a inhibitor, the most active developed Spindlin1 inhibitors were also tested over G9a and GLP to verify the selectivity profile of the $\mathbf{A} \mathbf{3 6 6}$ analogs. This resulted in the discovery of diverse selective compounds, among which 1s and 1t showed Spindlin1 activity in the nanomolar range and selectivity over G9a and GLP. Finally, future design hypotheses were suggested based on our findings.

Keywords: Spindlin1; structure-activity relationship (SAR); docking; molecular dynamics (MD) simulations; MM-GBSA

\section{Introduction}

Spindlin1 is a chromatin reader protein that plays an important role in epigenetic regulation through the recognition and interpretation of histone modifications. It consists of three Tudor domains, with the second domain binding to the H3K4me3 (H3 
trimethylated at lysine 4) mark [1-3]. More recently, its interaction with H4K20me3 (H4 trimethylated at lysine 20) was also described $[4,5]$. Since the binding of the latter histone mark displayed a weaker affinity than that of H3K4me3, it has been hypothesized that the H4K20me3 mark acts as a secondary binding partner for Spindlin1 [5]. Interestingly, the additional binding of the first domain with asymmetrically dimethylated arginine residues (Rme2a) has been shown to have different effects on the histone peptide affinity. Indeed, the H3K4me3R8me2a methylation pattern increases the affinity of H3K4me3, whereas H4K20me3R23me2a shows a lower binding affinity than H4K20me3 [3,5]. Very recently, another bivalent methylation pattern has been reported, namely H3K4me3K9me3/2, which has been described to enhance the histone binding affinity of H3K4me3 [6].

H3K4 is one of the most studied histone modifications, and it is well known to be tightly associated with the promoters of active genes that lead to transcriptional activation [7]. In contrast, H4K20 has been reported to have different functions based on the methylation states; H4K20me1 is associated with transcriptional activation, while H4K20me3 is linked with repression of transcription when present at promoters [8]. However, further studies are needed to investigate the biological relevance of the H4K20me3 mark and its functions correlated with Spindlin1 interactions.

Spindlin1 has been linked to several types of malignant tumors including ovarian cancer, breast cancer and triple negative breast cancer, non-small-cell lung cancers, liposarcoma, and very recently, liver cancer [9-14]. Moreover, it was found to be associated with drug-resistant breast cancer, as a study established that Spindlin1, which is negatively regulated by the miR-148/152 family, enhances Adriamycin resistance [15]. The literature also reports that Spindlin1 may play a role in tumorigenesis, and it was additionally suggested that Spindlin 1 is a proto-oncogene [16-20]. Some of the above-mentioned Spindlin1 functions have been related to diverse signaling pathways, such as Wnt ( $\beta$-catenin and TCF-4) [3,21], RET [13], PI3K/ Akt [10], and uL18-MDM2-p53 [19] pathways and SREBP1ctriggered FASN signaling [14]. In addition, Spindlin1 was found to control skeletal muscle development in mice and to play an important role in the first meiotic division of mammalian oocytes, thus providing potential links of Spindlin1 to human skeletal muscle diseases and human infertility, respectively $[22,23]$. Therefore, Spindlin1 inhibitors might be useful chemical tools to further investigate the physiological and pathological roles of this relatively new target.

To date, 15 crystal structures of Spindlin1 have been deposited in the RCSB Protein Data Bank (PDB) [24], both in apo form [25] and in complex with histone peptides [2,3,5], bivalent inhibitors, and small molecule inhibitors [26-28]. The crystal structure analysis and mutagenesis studies of some residues present in the first and second Tudor domains clearly indicated, as mentioned above, that the second domain is responsible for the recognition of the trimethylated lysine [2,3,5,13]. Specifically, four residues (Phe141, Trp151, Tyr170, and Tyr177) form the so-called aromatic cage in which the trimethylated lysine is embedded and stabilized by cation-pi interactions and hydrophobic van der Waals contacts.

In recent years, there has been growing interest in Spindlin1, which has led to the identification of several inhibitors blocking the Spindlin-H3K4me3 interaction. Through in silico studies coupled with in vitro testing, we identified novel Spindlin1 inhibitors active in the low $\mu \mathrm{M}$ range; as an example, compound Robaa-1k is illustrated in Figure 1 [29]. Using a screening platform, diverse compounds were identified, leading to the discovery of A366, a previously described G9a inhibitor ( $\mathrm{IC}_{50}: 3.3 \mathrm{nM}$, [30]), as a nanomolar Spindlin1 inhibitor ( $\mathrm{IC}_{50}: 182.6 \mathrm{nM}$, [31]) (Figure 1). Recently, applying the same approach to the screening of an epigenetic compound library, another hit was discovered, which was then simplified to a fragment-like inhibitor (MS31, Figure 1) [28]. Two different studies have identified bivalent inhibitors of Spindlin1 that simultaneously interact with its Tudor domain I and II (EML631 and VinSpinIn, Figure 1) [26,27]. 
<smiles>COc1cc2c(NCCCN3CCCC3)nc(=S)[nH]c2cc1OCc1ccccc1</smiles>

Robaa-1k<smiles>COc1c(CN)cc(CN)cc1OCCCN1Cc2ccccc2C1</smiles>

MS31<smiles>COc1cc2c(cc1OCCCN1CCCC1)N=C(N)C21CCC1</smiles>

A366<smiles>O=C(c1ccc(C(=O)N2CCC(CCN3CCCC3)CC2)c(Nc2ccc(OCN3CCCC3)cc2)c1)N1CCC(CCN2CCCC2)CC1</smiles>

EML631<smiles>CC1(C)C(N)=Nc2cc(OCCCN3Cc4ccc(OCCc5cn(CC(=O)N6CCC(CCN7CCCC7)CC6)nn5)cc4C3)c(OCC3CC3)cc21</smiles>

VinSpinln

Figure 1. Structures of reported Spindlin1 inhibitors.

Herein we report on the development of novel analogs of the A366 inhibitor. In an attempt to improve the activity of A366 and explore the structure-activity relationship (SAR), a congeneric series containing 21 A366 derivatives was synthesized and tested in vitro for their inhibitory activity against Spindlin 1 . This was accompanied by various computational approaches in order to investigate the possible binding mode of the synthesized derivatives, to explore the impact of the structural variations on the Spindlin1 inhibitory activity, and to identify a fast in silico method that could help to discriminate between active and inactive compounds within the A366 series. Additionally, to verify the selectivity of the new analogs, the most active compounds were also tested in vitro against G9a and GLP (G9a like protein).

\section{Results and Discussion}

In order to explore the SAR of A366 and to develop modified analogs, a congeneric series was synthesized and tested. This led to the generation of a dataset consisting of 21 compounds, 17 of which showed $\mathrm{IC}_{50}$ values in the range of $0.15-11.8 \mu \mathrm{M}$, two of which were found to be weakly active (Table $1, \mathbf{1 a}$ and $\mathbf{1 b}$ ), and three of which displayed a considerably decreased activity and were, thus, considered inactive (Table 1, 1c, 1d, 1e). Apart from A366 [31], 1f, and 1g [27], the other compounds are reported here for the first time as novel Spindlin1 inhibitors. The attained dataset was subsequently used for computational studies aimed to investigate the structural differences responsible for the activity of the compounds. Docking studies, molecular dynamics (MD) simulations, and molecular mechanics/generalized born surface area (MM-GBSA) rescoring calculations were performed using the Spindlin1 second domain as a binding site (PDB ID: 6I8Y). 
Table 1. Chemical structures and inhibitory activity of the A366 derivatives.

\begin{tabular}{|c|c|c|c|c|c|c|c|}
\hline Compounds & $\mathbf{R} 1$ & $\mathbf{R} 2$ & R3 & $\mathrm{R} 4$ & $\begin{array}{c}\text { Spin1 } \\
\mathrm{IC}_{50} \pm \mathrm{SD}(\mathrm{nM})^{[\mathrm{a}]}\end{array}$ & $\begin{array}{c}\text { G9a } \\
\text { IC }_{50}(\mathrm{nM})\end{array}$ & $\begin{array}{c}\text { GLP } \\
\mathrm{IC}_{50}(\mathrm{nM}){ }^{[\mathrm{b}]}\end{array}$ \\
\hline A366 & $\mathrm{Me}$ & $\mathrm{H}$ & & & $182.6 \pm 9.1[31]$ & $38^{[c]}$ & 51 \\
\hline $1 \mathrm{a}$ & Et & $\mathrm{OEt}$ & & & $70 \% @ 25 \mu \mathrm{M}^{[\mathrm{d}]}$ & & \\
\hline $1 b$ & $\mathrm{Me}$ & $\mathrm{H}$ & & & $81 \% @ 100 \mu \mathrm{M}^{[\mathrm{d}]}$ & & \\
\hline 1c & $\mathrm{Me}$ & $\mathrm{H}$ & & & $42 \% @ 50 \mu \mathrm{M}^{[\mathrm{d}]}$ & $>20,000$ & $>20,000$ \\
\hline 1d & $\mathrm{Me}$ & $\mathrm{H}$ & & & Inactive & & \\
\hline $1 e$ & $\mathrm{Me}$ & $\mathrm{H}$ & & & Inactive & & \\
\hline 1f & $\mathrm{Me}$ & $\mathrm{H}$ & & & $169 \pm 13$ & 431 & 334 \\
\hline $1 g$ & Et & $\mathrm{H}$ & & & $316 \pm 26$ & 243 & 2969 \\
\hline $1 \mathrm{~h}$ & $\mathrm{Me}$ & $\mathrm{CN}$ & & & $3900 \pm 700$ & & \\
\hline $1 \mathrm{i}$ & $\mathrm{Me}$ & $\mathrm{Cl}$ & & & $1600 \pm 200$ & $>20,000$ & $>20,000$ \\
\hline $\mathbf{1 j}$ & $\mathrm{Me}$ & $\mathrm{OMe}$ & & & $2500 \pm 200$ & & \\
\hline $1 \mathrm{k}$ & $\mathrm{Me}$ & Ac & & & $11,800 \pm 210$ & & \\
\hline 11 & Et & $\mathrm{H}$ & & & $567 \pm 35$ & & \\
\hline $1 \mathrm{~m}$ & 2-Pr & $\mathrm{H}$ & & & $3300 \pm 470$ & & \\
\hline 1n & $\mathrm{Me}$ & $\mathrm{H}$ & & & $255 \pm 35$ & 779 & 527 \\
\hline 10 & $\mathrm{Me}$ & $\mathrm{H}$ & & & $1210 \pm 90$ & $>20,000$ & $>20,000$ \\
\hline $1 p$ & $\mathrm{Me}$ & $\mathrm{H}$ & & $y$ & $157 \pm 12$ & 759 & 470 \\
\hline $1 \mathrm{q}$ & $\mathrm{Me}$ & $\mathrm{H}$ & & & $\begin{array}{c}\text { (R): } 470 \pm 27 \\
\text { (S): } 478 \pm 34 \\
\text { (rac): } 584 \pm 31\end{array}$ & (rac): 2321 & (rac): 4432 \\
\hline $1 \mathrm{r}$ & $\mathrm{Me}$ & $\mathrm{H}$ & & & $408 \pm 35$ & 3191 & 3185 \\
\hline $1 \mathrm{~s}$ & $\mathrm{Me}$ & $\mathrm{H}$ & & 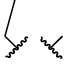 & $360 \pm 20$ & $>20,000$ & $>20,000$ \\
\hline $1 t$ & $\mathrm{Me}$ & $\mathrm{H}$ & & & $467 \pm 15$ & $>20,000$ & $>20,000$ \\
\hline
\end{tabular}


Table 1. Cont.

\begin{tabular}{|c|c|c|c|c|c|c|c|}
\hline Compounds & R1 & $\mathbf{R} 2$ & R3 & R4 & $\begin{array}{c}\text { Spin1 } \\
\mathrm{IC}_{50} \pm \mathrm{SD}(\mathrm{nM})^{[\mathrm{a}]}\end{array}$ & $\begin{array}{c}\text { G9a } \\
\text { IC }_{50}(\mathrm{nM})\end{array}$ & $\begin{array}{c}\text { GLP } \\
\mathrm{IC}_{50}(\mathrm{nM})\end{array}$ \\
\hline $1 u$ & $\mathrm{Me}$ & $\mathrm{H}$ & & & $1250 \pm 50$ & $>20,000$ & $>20,000$ \\
\hline
\end{tabular}

${ }^{\text {[a] }}$ Fluorescence polarization assay, based on competition to a Fluorescein-H3K4me3 peptide. ${ }^{[b]}$ Fluorescence based coupled enzyme assay, based on SAM-SAH conversion. For details, see experimental part. ${ }^{[c]}$ Reported $\mathrm{IC}_{50}$ G9a for A366: $3.3 \mathrm{nM}$ [30]. ${ }^{[\mathrm{d}]}$ If no IC 50 was obtained, inhibition is reported in percentage at specified concentration.

\subsection{Analysis of Spindlin1-A366 Complex}

The binding mode of A366 in the Spindlin1 second domain was first analyzed (Figure 2a,c) (PDB ID: 6I8Y [27]). This crystal structure shows a different shape of the binding pocket compared to the histones-bound crystal structures (e.g., PDB ID: 4H75 [2]). Indeed, A366 induces a flip of Phe141 in the aromatic cage; this conformational change of the phenylalanine side chain consequently opens the pocket and changes its shape and size. The protonated pyrrolidine moiety is embedded in the aromatic cage and undergoes cation-pi interactions with the surrounding amino acids. Notably, also the side chain of Trp151 is slightly adapted to better interact with A366. The amidine group is involved in salt bridge interactions with Asp184. The ligand binding mode is further stabilized by the formation of an intramolecular hydrogen bond between the methoxy group and the positively charged pyrrolidine-NH group. For comprehension and comparison, the crystal structure of Spindlin1 bound to the H3K4me3 histone peptide with a focus on the second domain is shown in Figure 2b,d (PDB ID: 4H75).

\subsection{Docking Studies}

Initially, our attention was focused on understanding the role of the spacer length (R3 group), since biological assays revealed that changing the linker from a propyl chain to ethyl/butyl chains results in a drop in activity (Table 1, compounds 1c, 1d, 1e). The compounds were hence docked into the crystal structure of Spindlin1 (PDB ID: 6I8Y) using Glide in the standard precision (SP) mode [32]. It is noteworthy that the used docking setup could successfully reproduce the experimentally determined binding mode and the interactions of A366 with an RMSD of $0.54 \AA$ (Supplementary Materials, Figure S1).

The obtained docking poses and docking scores (DS) of the inactive compounds were analyzed and compared to A366. Interestingly, the docking scores showed no substantial differences between the active and inactive compounds (DS range of inactive ligands: $-11.16 /-11.28 \mathrm{kcal} / \mathrm{mol}$; DS of A366: $-11.37 \mathrm{kcal} / \mathrm{mol}-$ Table S1). Docking of the inactive derivative 1e (Figure 3a), which bears a shorter ethoxy spacer as compared with A366, resulted in the same binding mode and showed the same interactions as observed for A366 (Figure 2c). On the other hand, the inactive derivative bearing a butoxy spacer, compound 1c, displayed a slightly different binding mode in which the amidine moiety is tilted towards Asp189 and establishes a salt bridge with it (Figure 3b). Nevertheless, the pyrrolidine ring of $\mathbf{1} \mathbf{c}$ is still properly located in the aromatic cage where it undergoes cation-pi interactions.

The main difference detected in the binding modes of all three inactive compounds (1c, 1d, and 1e) lies in the absence of the intramolecular hydrogen bond observed in the binding mode of $\mathbf{A 3 6 6}$. There is reason to believe that the formation of an intramolecular hydrogen bond plays an important role in stabilizing the active conformation. 
a
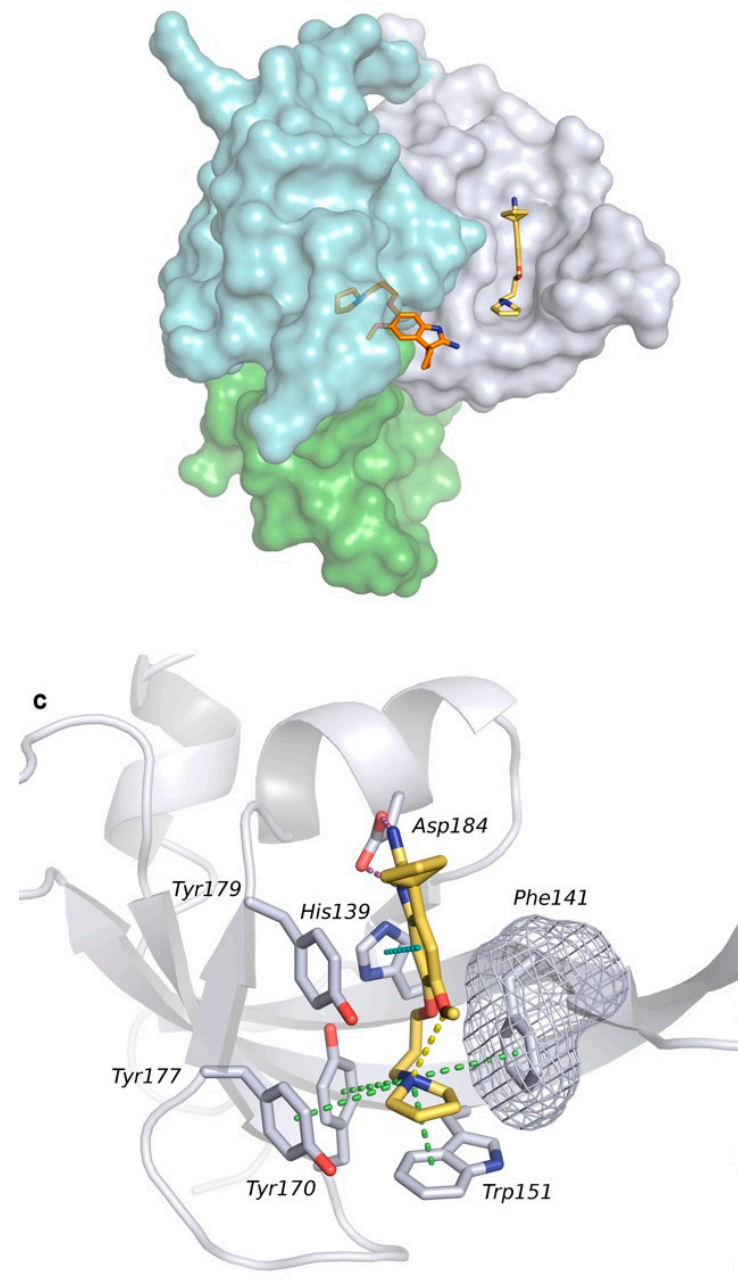

b
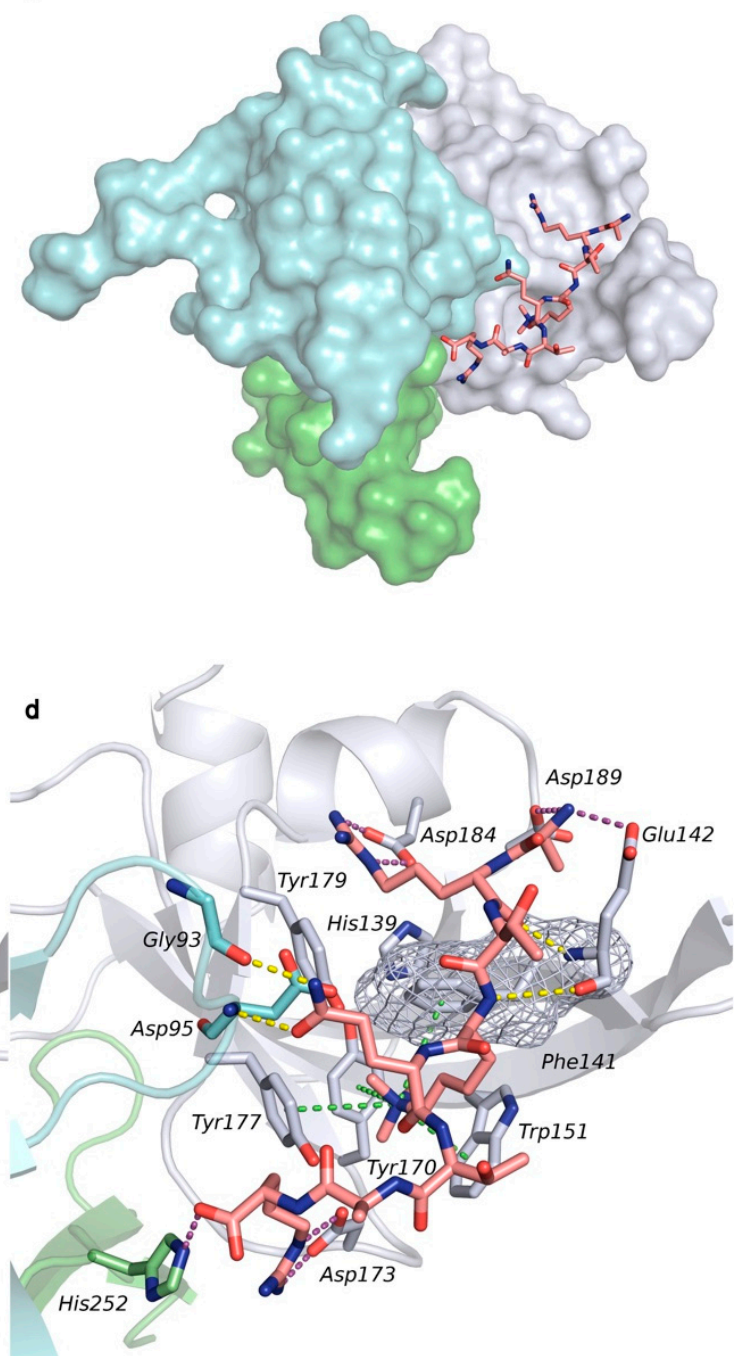

Figure 2. Crystal structures of Spindlin1 in complex with (a,c) A366 (PDB ID: 6I8Y) and (b,d) H3K4me3 (PDB ID: 4H75). $(\mathbf{a}, \mathbf{b})$ show the protein surfaces of the three Tudor domains, which are colored in cyan (domain I), white (domain II), and green (domain III). A366 bound to the first Spindlin1 domain is represented as orange sticks. (c,d) focus on domain II and the binding interactions of $\mathbf{A} 366$ (yellow sticks) and H3K4me3 histone peptide (pink sticks). Only the side chains of the surrounding residues are shown for clarity, and they are displayed as sticks; for residues Gly93, Asp95, and Glu142, the main chains are shown. Phe141 is also depicted by the surface mesh. Hydrogen bonds are represented as dashed yellow lines, cation-pi interactions as dashed green lines, pi-pi stacking interactions as dashed cyan lines, and salt bridges as dashed magenta lines.

\subsection{Molecular Dynamic Simulations}

Given the poor understanding of the compounds' inactivity by docking scores and predicted binding mode, the next step was the investigation of these ligands by means of MD simulations. The obtained docking poses of A366 and two inactive compounds (1e and 1c) were subjected to $200 \mathrm{~ns}$ MD simulations using Amber18 package [33] to gain insight into the stability of the predicted binding modes and protein-ligand interactions. 


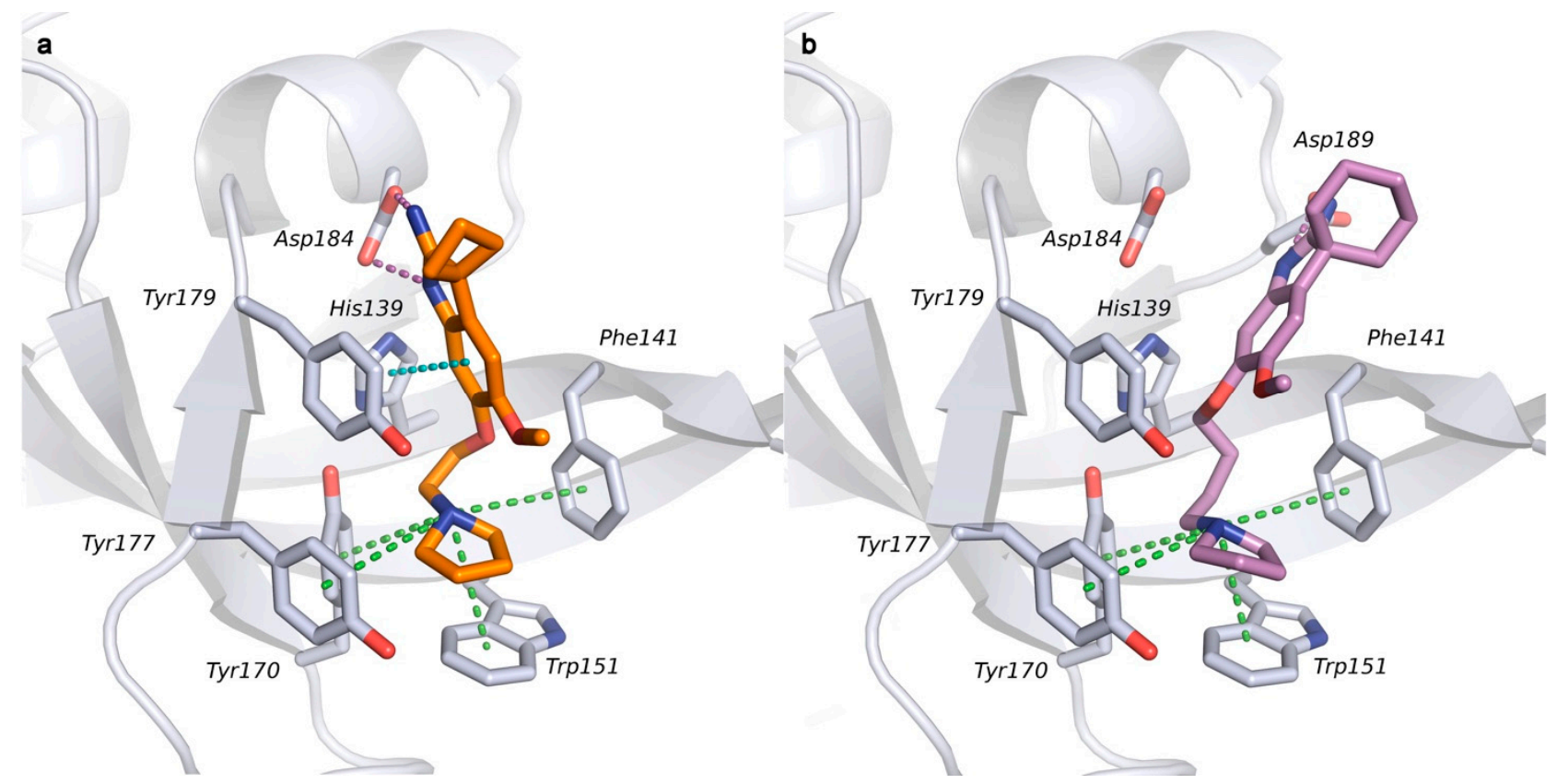

Figure 3. Docking poses of 1e (a) and 1c (b) in the Spindlin1 second domain (PDB ID: 6I8Y). Docking poses and side chains of the surrounding residues are shown as sticks. Binding interactions are represented with dashed lines colored in green (cation-pi), cyan (pi-pi stacking), and magenta (salt bridge).

The analysis of the MD simulation performed for A366 indicated a stable binding mode, where the interactions observed in the crystal structure (Figure 2c) are preserved throughout the simulation. It is noteworthy that the intramolecular hydrogen bond between the methoxy group and the pyrrolidine- $\mathrm{N}^{+} \mathrm{H}$ was also maintained throughout the MD simulation. Meanwhile, for both inactive derivatives, 1c and 1e, this intramolecular hydrogen bond was barely observed during the MD simulation. Figure 4a shows the root-mean-square deviation (RMSD) plot, while the occupancy rates of the most important interactions are reported in the heat map (Figure 5).

Compound 1c, despite its inactivity, was found to be relatively stable throughout the MD simulation in terms of RMSD values, albeit less stable than A366 (Figure 4b). Although the obtained docking pose of $1 \mathrm{c}$ shows an interaction between the amidine moiety of the ligand and Asp189 (Figure 3b), this interaction is lost during the MD simulation (occupancy $2 \%$ ). Indeed, directly at the beginning of the MD simulation, the aminoindole moiety moves so that its amidine group establishes a salt-bridge with the side chain of Asp184, which is maintained for ca. $94 \%$ of the simulation time. Apart from the aminoindole group, small fluctuations of the ligand are observed, which mainly affect the pyrrolidine moiety and, consequently, the formation of cation-pi interactions. This was highlighted by the decreased occupancy of these interactions during the simulation (Figure 5). In fact, with the exception of the cation-pi interactions established with Trp151, the occupancies of the other aromatic interactions are dramatically decreased, pointing out the important role of these interactions for the activity.

On the other hand, the instability of the binding mode obtained for 1e could give a plausible explanation for its inactivity (Figure 4c). Indeed, diverse binding conformations were detected during the MD simulation (Supplementary Materials, Figure S2), all of which seem to be unstable, as demonstrated also by the low occupancy rates of the observed interactions, as detailed in Figure 5. The salt bridge between the amidine group and Asp184 (occupancy rate 31\%) is completely lost after 70 ns simulation time (Supplementary Materials, Figure S3). Moreover, the analysis of the cation-pi interactions underlined that even though the positively charged pyrrolidine moiety is located in the aromatic cage, its position is not optimal for establishing aromatic interactions with all amino acids that form 
the cage. This can be seen in Figure 5, specifically, in the low occupancy rates obtained for the interactions with Phe141 and Tyr177 (54.1\% and 55.5\%, respectively).

a

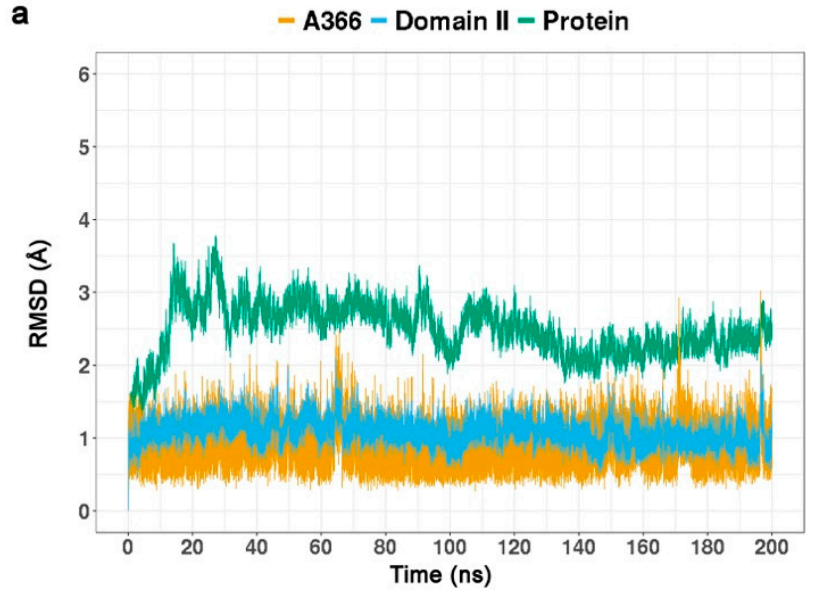

C

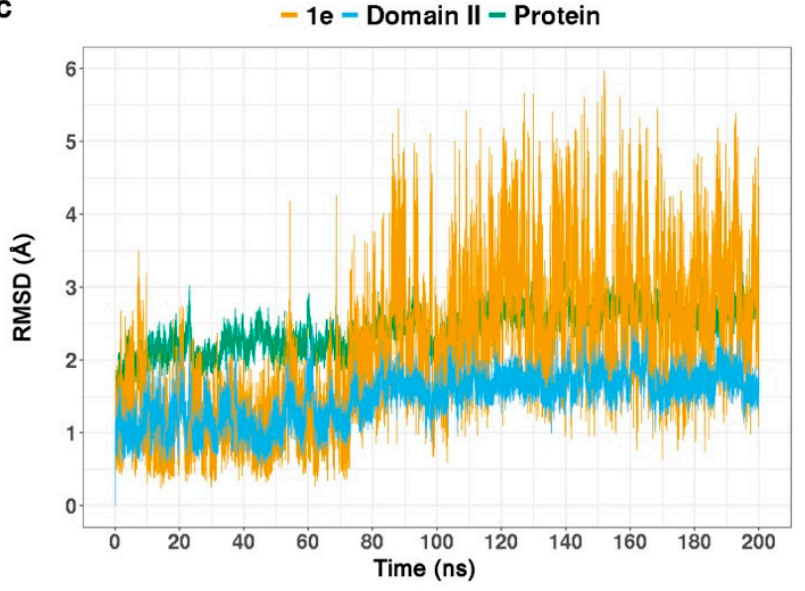

b

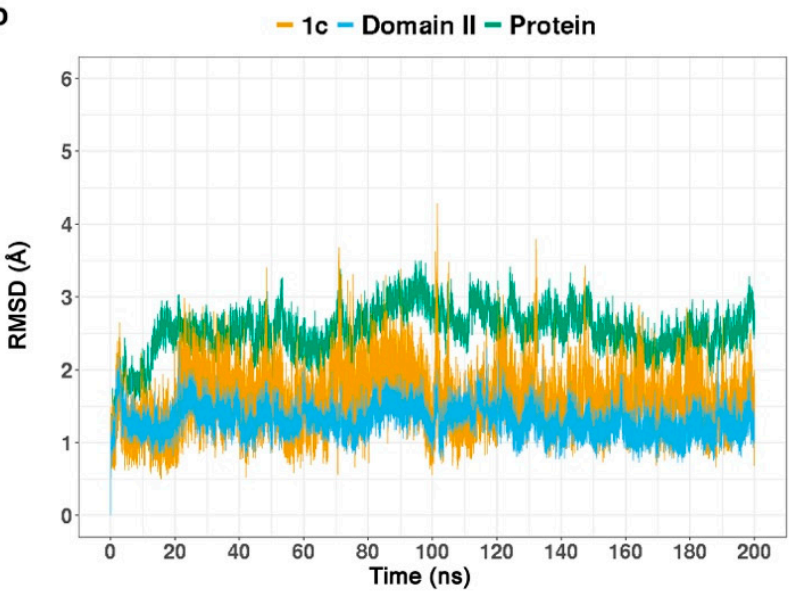

Figure 4. Analysis of $200 \mathrm{~ns}$ MD simulations of reference ligand A366 and inactive compounds 1c and 1e. Root mean square deviation (RMSD) plots of the systems with (a) A366, (b) 1c, and (c) 1e.

In conclusion, based on the MD simulations analysis of these three compounds, we could confirm the assumption that the spacer length is important for stabilizing the binding pose and optimizing the binding interactions. The finding shows that the linker chain can affect not only the presence of intramolecular hydrogen bond, but also the optimal location of the pyrrolidine ring in the pocket and generally the binding conformation, which in turn influences the formation of cation-pi interactions with the aromatic cage as well as the interactions of the amidine moiety with Asp184.

\subsection{Re-Scoring Using Prime MM-GBSA}

Although the analysis of the MD simulations was able to rationalize the difference in the compounds' activity, this approach is time-consuming for a bigger dataset. Thus, the synthesis of the new derivatives was coupled with docking and MM-GBSA rescoring to find a fast approach that could help in discriminating active from inactive compounds of the same congeneric series. The structural differences between the active and inactive compounds lie in the linker chain, where the actives bear a propyl chain, whereas the inactive compounds bear either an ethyl or a butyl chain (Table 1, compounds 1c, 1d, and 1e). Only ligands with determined $\mathrm{IC}_{50}$ values were used as actives. 
a

\begin{tabular}{r|c:ccc} 
& A366 & 1c & 1e \\
\hline \multirow{2}{*}{ Cation-pi } & Trp151 & 99.9 & 99.9 & 99.5 \\
& Tyr170 & 86.2 & 33.5 & 77.9 \\
& Tyr177 & 89.0 & 41.9 & 55.5 \\
\cline { 1 - 3 } Salt bridge & Asp184 & 97.0 & 94.1 & 31.0 \\
& Asp189 & 0.1 & 2.0 & 2.7 \\
\cline { 2 - 3 } Intra H-bond & 69.1 & 1.6 & 11.3 \\
\hline
\end{tabular}

$0 \%$ b

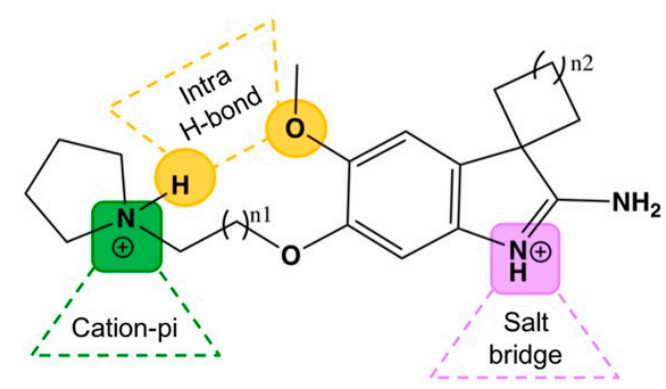

A366: $\mathrm{n} 1=2, \mathrm{n} 2=1$

1c: $\mathrm{n} 1=3, \mathrm{n} 2=3$

1e: $\mathrm{n} 1=1, \mathrm{n} 2=1$

Figure 5. Summary of the binding interactions identified during $200 \mathrm{~ns}$ MD simulations of the reference ligand A366 and the inactive compounds 1c and 1e. The binding poses were obtained from docking into Spindlin1 second domain binding site (PDB ID: 6I8Y). (a) Heat map showing the occupancy rates of the observed interactions. (b) Common structure of the compounds highlighting the atoms involved in the interactions.

First, since from the above-described docking studies and MD simulations analysis the key role of the intramolecular hydrogen bond was evident, its contribution was incorporated into the docking scores. This was done by allowing the option "reward intramolecular hydrogen bonds" in Glide [32]; in this way, the compounds that exhibit these intramolecular interactions pay a smaller entropic penalty upon binding, which affects the final docking scores (DS_Intra-H-bonds). For the analysis of the results, box-plots were generated showing the distribution of docking scores without the "reward intramolecular hydrogen bonds" option (DS) and DS_Intra-H-bonds attained for our active and inactive ligands. As illustrated in Figure 6a, the DS values retrieved for active and inactive compounds are distributed in the same range, which clearly demonstrates that this scoring function is not able to discriminate between active and inactive compounds. On the other hand, a small improvement is observed when DS_Intra-H-bonds values are evaluated (Figure 6b). However, the difference between active and inactive ligands is still too small to be considered reliable, as highlighted by the mean values $(-12.0 \mathrm{kcal} / \mathrm{mol}$ for the actives and $-11.2 \mathrm{kcal} / \mathrm{mol}$ for the inactive compounds).
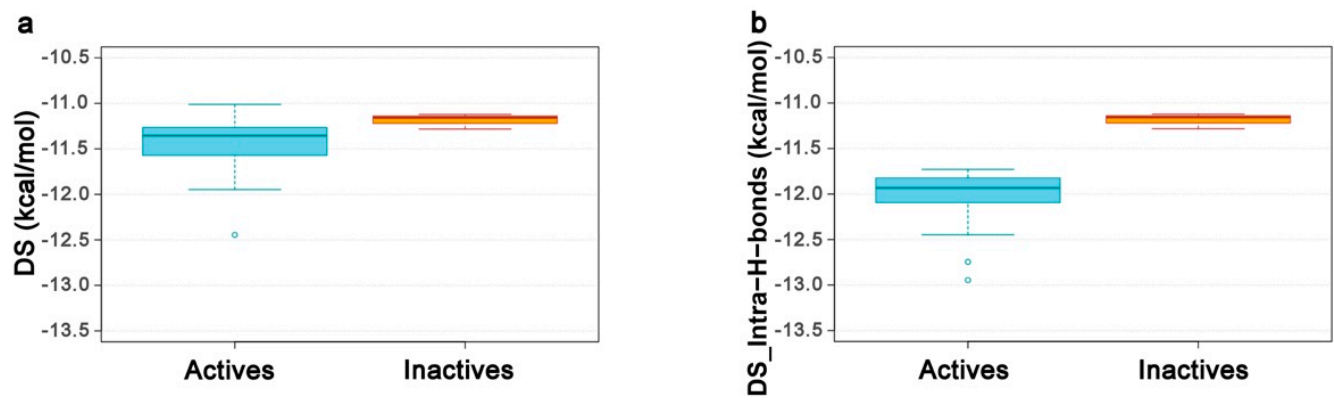

Figure 6. Box-plots showing docking scores distribution obtained for active and inactive compounds using glide standard precision (a) without intramolecular hydrogen bonds contribution and (b) rewarding intramolecular hydrogen bonds.

Owing to the low discriminatory power of the glide docking scores (DS and DS_IntraH-bonds), the docking poses were post-processed using more accurate binding free energy (BFE) calculations with Prime MM-GBSA. Diverse settings were tested; here, we report the results obtained when only the ligands were relaxed, and when also the protein residues within $3 \AA$ Af the ligands were treated as flexible. The computed binding affinity values 
were visualized by box-plots, seen in Figure 7. Interestingly, the re-scoring methods were able to successfully discriminate between active and inactive compounds. The calculated BFE values retrieved for the active compounds were more favorable than those of the inactive compounds, and good discrimination ranges were found, as emphasized by the significant gaps between the mean values (MM-GBSA $\Delta \mathrm{G}_{\mathrm{bind}}$ mean values of $-76.3 \mathrm{kcal} / \mathrm{mol}$ (actives) and $-60.35 \mathrm{kcal} / \mathrm{mol}$ (inactives); MM-GBSA $\Delta \mathrm{G}_{\mathrm{bind}}-3 \AA$ mean values of $-76.3 \mathrm{kcal} / \mathrm{mol}$ (actives) and $-62.6 \mathrm{kcal} / \mathrm{mol}$ (inactives)).

a

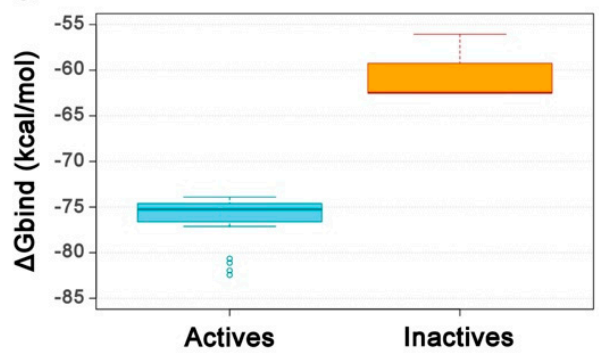

b

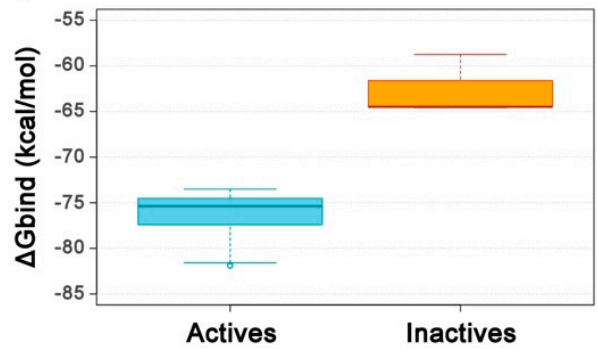

Figure 7. Box-plots showing $\Delta \mathrm{G}_{\text {bind }}$ distribution obtained for active and inactive compounds using Prime MM-GBSA. (a) Default setting, only ligands are relaxed (b) also protein residues around $3 \AA$ from the ligands are treated as flexible.

The two re-scoring methods performed equally well from an efficiency perspective, but differently for the computation time. The CPU time required for the calculation of one inhibitor was $0.6 \mathrm{~min}$ when only the ligand was treated as flexible, but $1.7 \mathrm{~min}$ when the protein residues were included (MM-GBSA-3 $\AA$ ). Given the promising findings, this developed protocol can be applied to guide further optimization steps in order to prioritize compounds for synthesis and biological characterization.

\subsection{Structure-Activity Relationships}

Once the role of the spacer was clear, we were interested in exploring the effect of other substitutions on the ligand's inhibitory activity. The synthesized dataset of A366 derivatives encompassed compounds bearing different spiro and dialkyl substituents at position-3 of the indole ring (R4), different substituents at the pyrrolidine ring, as well as varying groups at the $\mathrm{R} 1$ and $\mathrm{R} 2$ positions of the indole ring.

To investigate the influence of these modifications on the Spindlin1 inhibitory activity, the fluorescence polarization assay established earlier [31] was used to determine the potency of the compounds. Furthermore, the most active Spindlin1 inhibitors were tested in vitro against G9a and GLP to analyze their selectivity profile.

The biological assays revealed that the introduction of a substituent on R2 position led to a significant decrease in the Spindlin1 inhibitory activity as observed for $\mathbf{1 a}, \mathbf{1 h}, \mathbf{1} \mathbf{i}$, $\mathbf{1 j}$, and $\mathbf{1 k}$ ( $\mathrm{IC}_{50}$ values: $1.6-11.8 \mu \mathrm{M}$ ) when compared to the parent compound $\mathrm{A} 36 \mathbf{6}\left(\mathrm{IC}_{50}\right.$ $182.6 \mathrm{nM})$. Meanwhile, the replacement of the methoxy group at R1 with ethoxy $(\mathbf{1 g}, \mathbf{1 1})$ and isopropoxy $(\mathbf{1 m})$ groups showed different impacts on the activity of the compounds. The ethoxy derivatives showed only a two-fold decrease in the inhibitory activity, as observed when comparing $\mathbf{1 g}$ and $\mathbf{1 1}$ with $\mathbf{1 f}$ and $\mathbf{1 n}$, respectively. On the other hand, the isopropoxy derivative $1 \mathrm{~m}$ displayed a significant decrease in activity with an $\mathrm{IC}_{50}$ value in the micromolar range.

Modifications of the spiro moiety (R4) had no major impact on the Spindlin1 inhibitory activity of the compounds; cyclobutyl, -pentyl, and -hexyl rings as well as the dimethyl analogues (A366, 1f, 1n, 1p) were all well tolerated. Meanwhile, the 3-ethyl3-methyl analogue $1 \mathrm{r}\left(\mathrm{IC}_{50} 408 \pm 35 \mathrm{nM}\right)$ displayed only a two-fold decrease in activity as compared to A366. However, no selectivity was detected for these compounds. Among the attempted R4-modifications, the major impact was observed with compound 10 bearing the geminal diethyl group, which exhibited a significantly decreased activity for Spindlin1 ( $\mathrm{IC}_{50}: 1.21 \pm 0.09 \mu \mathrm{M}$ ). Interestingly, this was coupled with an increase in 
selectivity for Spindlin1 over G9a and GLP (G9a IC $50>20 \mu \mathrm{M}$, GLP IC $50>20 \mu \mathrm{M}$ ). Noteworthy, the analogous derivative with dimethyl groups, 1p, which showed an activity comparable to $\mathbf{A} 366$ and was one of the most active Spindlin1 inhibitors of our series (Spindlin1 IC $\mathrm{I}_{50}: 157 \pm 12 \mathrm{nM}$ ), showed no selectivity over G9a and GLP (G9a IC 50 : $759 \mathrm{nM}$, GLP IC $_{50}: 470 \mathrm{nM}$ ). The decrease in the Spindlin1 activity going from geminal dimethyl (1p) to 3-ethyl-3-methyl (1r) and ultimately to geminal diethyl (1o) is inversely related to the number of rotatable bonds and can, thus, be associated with the loss of conformational entropy of the compounds.

Given the important role of the pyrrolidine moiety in the binding pocket, it was not surprising that its modifications could impact the activity of the compounds. Notably, the replacement of the pyrrolidine ring in $\mathbf{1 f}$ by a 3-hydroxypyrrolidine moiety (1q) only led to three-fold decrease in the Spindlin1 activity $\left(\mathrm{IC}_{50}\right.$ values of $169 \mathrm{nM}$ and $584 \mathrm{nM}$, respectively). The docking studies of 1q predicted binding modes in which the hydroxy group is engaged in a hydrogen bond interaction with Tyr177 (Figure 8a). On the other hand, the replacement of the pyrrolidine ring by an isoindoline moiety led to compound 1s, which showed strong inhibition of Spindlin1 ( $\mathrm{IC}_{50}: 360 \pm 20 \mathrm{nM}$ ) combined with selectivity (G9a IC $50>20 \mu \mathrm{M}$, GLP IC $_{50}>20 \mu \mathrm{M}$ ). Our docking studies in the Spindlin1 second domain binding site reveal that the aromatic ring of the isoindoline moiety can further stabilize the ligand through the formation of additional pi-pi stacking interactions with Phe141 and Trp151 in the aromatic cage (Figure 8b). To further explore the binding mode of compound 1s, the obtained docking pose was subjected to $100 \mathrm{~ns}$ MD simulation. The analysis showed that the predicted binding mode is stable, and the key interactions (cation-pi, salt bridge, and intramolecular hydrogen bond) are conserved during the simulation time. However, the occupancy rates of the additional pi-pi stacking highlighted that such interactions are less preserved throughout the MD simulation (Figure S4).

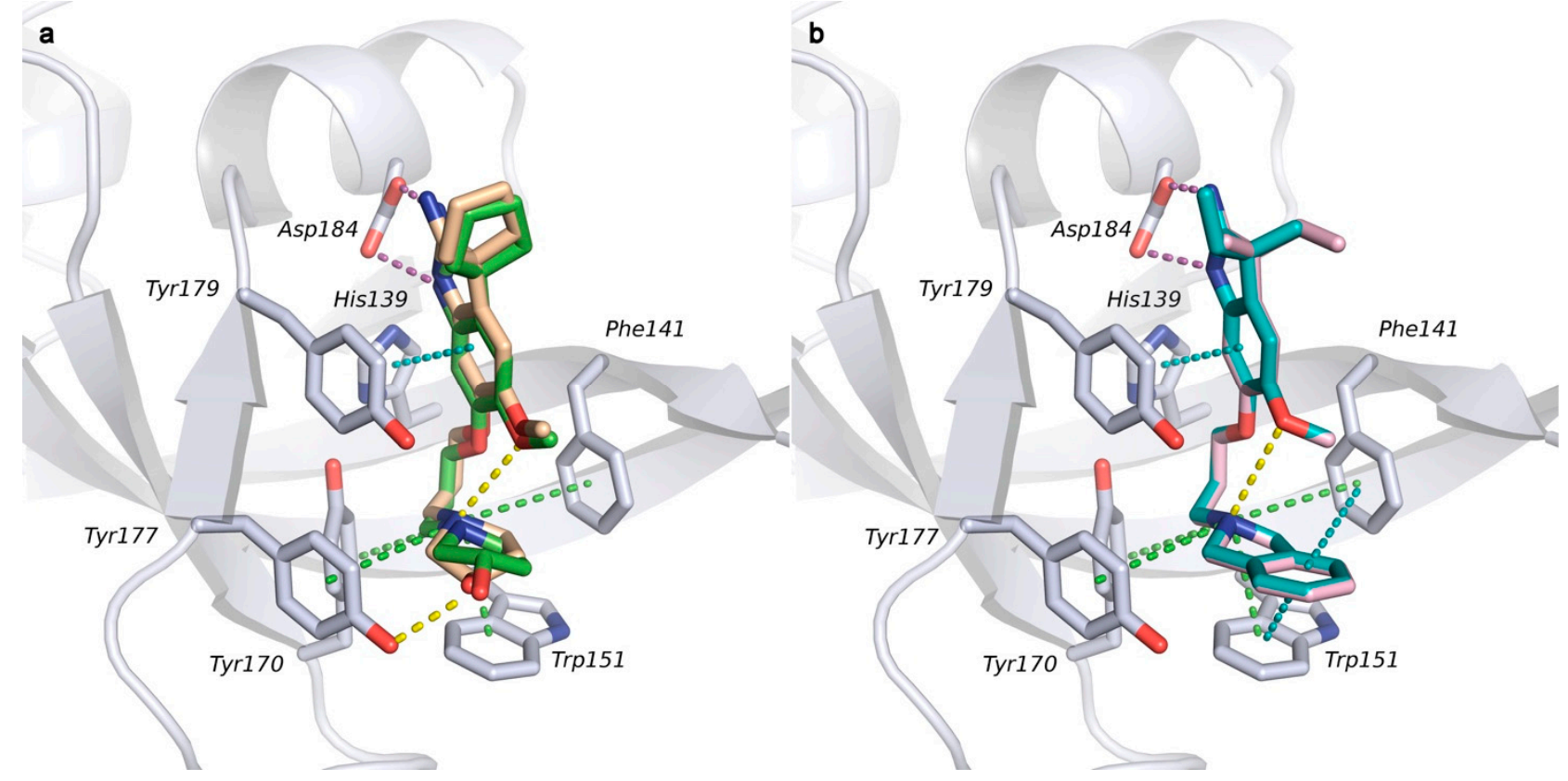

Figure 8. Predicted binding modes of some derivatives in the Spindlin1 second domain binding site (PDB ID: 6I8Y). (a) Compound 1q (S, green; R, beige). (b) Compound 1s (S, cyan; R, light pink). Docking poses and side chains of the surrounding residues are shown as sticks. Binding interactions are represented with dashed lines colored in yellow (hydrogen bond), green (cation-pi), cyan (pi-pi stacking), and magenta (salt bridge).

A good selectivity profile was also observed for compound $\mathbf{1 t}$, which bears a 3methylpyrrolidine moiety and displays a distinct inhibition of Spindlin1 $\left(\mathrm{IC}_{50}: 467 \pm 15 \mathrm{nM}\right)$ coupled with selectivity over G9a (G9a IC $50>20 \mu \mathrm{M}$, GLP IC $50>20 \mu \mathrm{M})$. In conclusion, the 
obtained data underlined that specific substitutions on the pyrrolidine moiety by small substituents like 3-methyl and 3-hydroxymethyl groups as well as replacement of the pyrollidine ring by an isoindoline moiety led to a significant drop in the activity for G9a and GLP whilst maintaining the potent inhibitory activity against Spindlin1. These substitution patterns can hence be used to design selective compounds.

\subsection{Synthesis}

All compounds were synthesized from easily accessible starting materials such as guaiacol derivatives 2 (Scheme 1, Route A) or vanillin derivatives 3 (Scheme 1, Route B). In the first route, bromination of $\mathbf{2}$ was achieved in a regioselective manner at low temperatures in order to obtain phenols 7 , which were directly benzylated and conveniently purified by recrystallization, resulting in excellent yields of intermediates 8 . The organolithium species resulting from subsequent bromine-lithium exchange were then added to a variety of ketone derivatives resulting in benzyl alcohols 5. Mediated by a Lewis acid activation, benzyl cyanides 10 were obtained by a substitution reaction. After benzylation of their phenol moiety, the second route was initiated by the reduction of aldehydes 6 with lithium aluminum hydride. Benzyl alcohols 7 were generally obtained in excellent yields and could be transformed into benzyl cyanides 9 via two successive substitution steps using first concentrated hydrochloric acid and then potassium cyanide as nucleophile sources. Double alkylation in benzyl position under basic conditions led to either symmetrically dialkylated or cycloalkylated benzyl cyanides $\mathbf{1 0}$, which were intermediates common to both synthetic routes. Nitration was then performed at low temperatures and under mild conditions; the lower yields of the desired regioisomers $\mathbf{1 1}$ resulted from poor regioselectivity for certain derivatives. Deprotection of the phenol moiety was selectively achieved using aluminum chloride. Typical hydrogenation conditions involving palladium on carbon as the catalyst partially resulted in a reduction of the nitro group for several derivatives, leading to a significant loss of desired materials 12. Alkylation of the unprotected phenol moiety was performed either directly with 1-(3-chloropropyl)pyrrolidine under basic conditions or as a two-step sequence including first a terminal dihaloalkane with the desired spacer length and then the $N$-heterocyclic compound of choice. The final step consisted of reducing compounds 13 using zinc dust in acetic acid, delivering the 2-aminoindole derivatives 1a-u resulting from direct ring closure under the chosen conditions.

Route A:

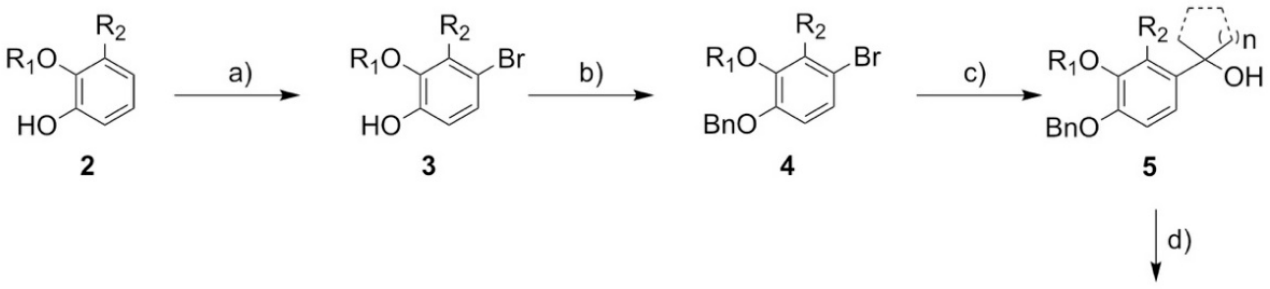

Route B:

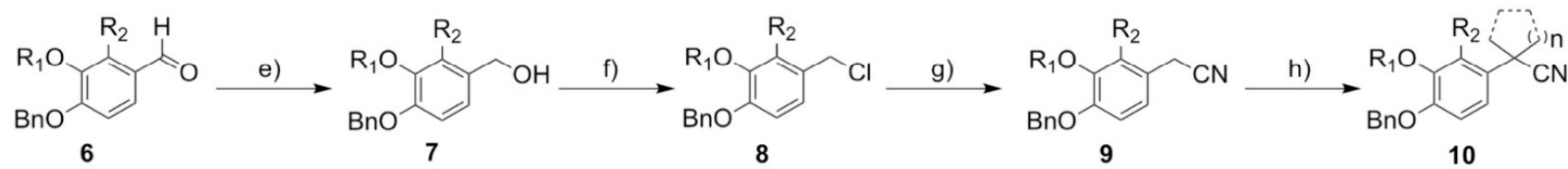

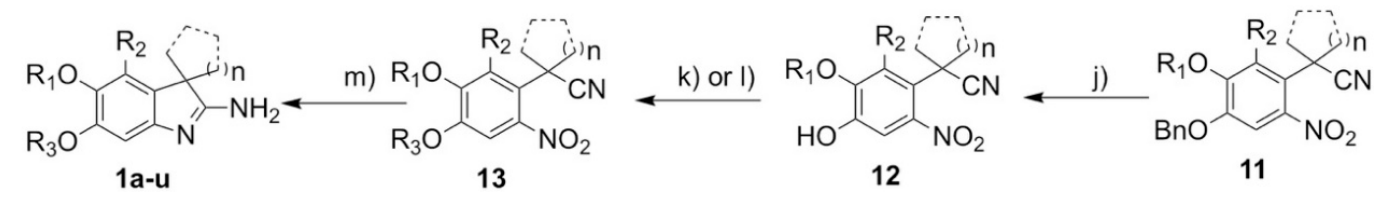

Scheme 1. Reagents and conditions: (a) $\mathrm{Br}_{2}$ (1.1 eq), $\mathrm{DCM},-20$ to $0{ }^{\circ} \mathrm{C}, 1 \mathrm{~h}$; (b) $\mathrm{K}_{2} \mathrm{CO}_{3}(1.2 \mathrm{eq}), \mathrm{BnBr}(1.2 \mathrm{eq}), \mathrm{DMF}, 90{ }^{\circ} \mathrm{C}$, 
$2 \mathrm{~h}, 91-95 \%$ over two steps; (c) $n$-BuLi (1.1 eq); ketone (1.1 eq), THF, $-78{ }^{\circ} \mathrm{C}$ to $\mathrm{rt}, 1 \mathrm{~h}, 44-70 \%$; (d) TMSCN (3.0 eq); $\mathrm{SnCl}_{4}$ (1.0 eq), DCM, $-78{ }^{\circ} \mathrm{C}$ to rt, $0.5 \mathrm{~h}, 39-90 \%$; (e) $\mathrm{LiAlH}_{4}$ (1.5 eq), THF, $0{ }^{\circ} \mathrm{C}$ to rt, $4 \mathrm{~h}, 94-97 \%$; (f) conc. $\mathrm{HCl}(15 \mathrm{eq}), \mathrm{Et}_{2} \mathrm{O}$, $0{ }^{\circ} \mathrm{C}$ to rt, $30 \mathrm{~min}, 93-98 \%$; (g) KCN (1.1 eq), NaI (0.5 eq), DMF, $80{ }^{\circ} \mathrm{C}, 17 \mathrm{~h}, 74-79 \%$; (h) $\mathrm{KOH}(7 \mathrm{eq}), 1, n$-dibromo- $n$-alkane (1.0 eq), TBAB ( $1 \mathrm{~mol} \%), \mathrm{PhMe} / \mathrm{H}_{2} \mathrm{O}$, reflux, $1.0 \mathrm{~h}, 56-63 \%$; or $\mathrm{NaH}(2.5 \mathrm{eq})$; iodomethane (4 eq), THF, reflux, $16 \mathrm{~h}, 90 \%$; (i) $\mathrm{HNO}_{3}$ (1.5 eq), $\mathrm{Ac}_{2} \mathrm{O} / \mathrm{AcOH}, 0{ }^{\circ} \mathrm{C}, 1.0$ h, 56-92\%; (j) $\mathrm{AlCl}_{3}$ (3.0 eq), DCM, $0{ }^{\circ} \mathrm{C}, 1$ h, 80\%-quant.; (k) $\mathrm{K}_{2} \mathrm{CO}_{3}(1.4 \mathrm{eq}), \mathrm{NaI}$ (0.2 eq), 1-(3-chloropropyl)pyrrolidine (1.2 eq), DMF, $80{ }^{\circ} \mathrm{C}, 1.5 \mathrm{~h}, 67-92 \%$; (1) 1 . NaH (1.2 eq); $1, n$-dihalo- $n$-alkane (1.5 eq), DMF, 0 to $100{ }^{\circ} \mathrm{C}, 2 \mathrm{~h}, 2$. $N$-heterocyclic compound (2 to $\left.10 \mathrm{eq}\right), \mathrm{DMF}, 70{ }^{\circ} \mathrm{C}, 2-3 \mathrm{~h}, 30-86 \%$ over two steps; (m) Zn (10-15 eq), HOAc, $85^{\circ} \mathrm{C}, 1 \mathrm{~h}, 61-99 \%$.

\section{Materials and Methods}

\subsection{Synthesis}

\subsubsection{General Methods and Materials}

Reagents and solvents were obtained from commercial sources and used without any further purification. Column chromatography was accomplished using MACHEREYNAGEL silica gel $60^{\circledR}(230-400$ mesh). Thin layer chromatography was performed on aluminum plates pre-coated with silica gel (MERCK, 60 F254) unless specified. TLCs were visualized by UV fluorescence $\left(\lambda_{\max }=254 / 320 \mathrm{~nm}\right)$ and/or by staining.

NMR spectra were acquired on a BRUKER Avance 400 spectrometer $(400 \mathrm{MHz}$ and $100.6 \mathrm{MHz}$ for $1 \mathrm{H}$ and $13 \mathrm{C}$ respectively) or a Bruker $500 \mathrm{DRX}$ NMR spectrometer with TBI probe head (499.6 and $125.6 \mathrm{MHz}$ for $1 \mathrm{H}$ and $13 \mathrm{C}$ respectively) at a temperature of $303 \mathrm{~K}$ unless specified. Chemical shifts are reported in parts per million (ppm) relative to residual solvent.

Data for $1 \mathrm{H}$ NMR are described as follows: chemical shift ( $\delta$ in ppm), multiplicity ( $\mathrm{s}$, singlet; $\mathrm{d}$, doublet; $\mathrm{t}$, triplet; q, quartet; quin, quintuplet; hept, heptuplet; $\mathrm{m}$, multiplet; $\mathrm{br}$, broad signal), coupling constant $(\mathrm{Hz})$, and integration. Data for 13C NMR are described in terms of chemical shift ( $\delta$ in ppm).

HR-MS were obtained on a THERMO SCIENTIFIC Advantage and a THERMO SCIENTIFIC Exactive instrument (APCI/MeOH: spray voltage $4-5 \mathrm{kV}$, ion transfer tube: $250-300{ }^{\circ} \mathrm{C}$, vaporizer: $300-400{ }^{\circ} \mathrm{C}$ ).

Optical rotation of chiral compounds was determined on a PERKIN-ELMER PE 241 apparatus and transformed for a given temperature according to the following formula (Equation (1)):

$$
[\alpha]_{\mathrm{D}}^{\mathrm{T}}=\frac{\alpha \times 100}{\mathrm{c} \times 1}
$$

$\alpha$ : measured value for optical rotation; c: concentration in $\mathrm{g} / 100 \mathrm{~mL} ; \mathrm{l}$ : length of the cuvette in $\mathrm{dm}$; $\mathrm{T}$ : temperature in ${ }^{\circ} \mathrm{C}$.

\subsubsection{Synthesis and Analytical Data}

Detailed synthetic route for each final derivative as well as syntheses and data for intermediary compounds are given in the Supplementary Materials.

\subsection{Biological Assays}

\subsubsection{Fluorescence Polarization Assay}

Fluorescence polarization assays were carried out as described before [31]. The inhibitors were diluted in assay buffer (25 mM HEPES pH 7.5, NaCl $100 \mathrm{mM}, 0.05 \%$ CHAPS, $1 \mathrm{mg} / \mathrm{mL} \mathrm{BSA}$ ) in a serial dilution of 12 inhibitor concentrations with equal DMSO concentrations, and added in a 384-well black non-binding microplate (Greiner Bio-One) to mixtures of H3(1-14)K4me3-Fluorescein (Peptide Specialty Laboratories) and HisSpindlin1(49-262), with final concentrations of 10 and $100 \mathrm{nM}$, respectively. After $30 \mathrm{~min}$ incubation at room temperature, the fluorescence polarization (Ex480, Em535) was measured using an Envision Plate reader (PerkinElmer). For the controls, a buffer solution with the same DMSO concentration as the inhibitor solutions was added to the plate instead of the inhibitor solution, the negative control contained the H3(1-14)K4me3-Fluorescein 
without Spindlin1. The blank control contained buffer solution with DMSO. Every inhibitor concentration and the controls were done in triplicates. Fluorescence polarization values $(\mathrm{mP})$ were determined after blank correction as $\frac{I_{S}-G \times I_{P}}{I_{S}+G \times I_{P}} \times 1000$, where $\mathrm{G}$ is a device-specific factor and IS and IP are the fluorescence intensities of the $S$ and $P$ plane, respectively. Inhibition values were then calculated as $I=100 \% \times\left(1-\left(\frac{P_{I}-P_{\text {neg }}}{P_{\text {pos }}-P_{\text {neg }}}\right)\right)$, with PI, $P_{\text {neg }}$, and $P_{\text {pos }}$ being the fluorescence polarization results of the ligand solution, the negative control, and the positive control, respectively. Curve fitting was carried out using GraphPad Prism 7, applying a non-linear sigmoidal fit with a variable slope. Inhibition curves used for $\mathrm{IC}_{50}$ determination are reported in the Supplementary Materials (Figure S5).

\subsubsection{Reagents for Histone Methyltransferase Assays}

H3 peptide (1-25) is purchased from Anaspec (Cat. NO. AS-61703), thiol fluorescent probe IV is purchased from Millipore-Sigma (Cat. NO. 595504), SAH (S-Adenosyl-Lhomocysteine) is purchased from NEB (Cat. NO. B9003S), and adenosine deaminase (ADA) is purchased from Sigma (Cat. NO. 10102105001).

\subsubsection{Expression and Purification of G9a and GLP and SAHH}

Catalytic domains of Human G9a (913-1193) and GLP (982-1266) were cloned, expressed, and purified as previously described [34]. Full length of S. solfataricus Sadenosylhomocysteine hydrolase (SAHH) was cloned, expressed, and purified using the published protocol [35].

\subsubsection{Coupled Enzyme Fluorescent Histone Methyltransferase Assay}

The assay was performed using the previously published protocol [35]. Tested compounds were pre-dissolved in assay buffer (20 mM HEPES pH 7.5, $50 \mathrm{mM} \mathrm{NaCl}, 0.01 \%$ Triton X-100, $3 \mathrm{mM} \mathrm{MgCl} 2,0.1 \mathrm{mg} / \mathrm{mL}$ BSA) with $5 \mu \mathrm{M}$ of purified SAHH, 1 unit of ADA, $10 \mu \mathrm{M}$ of $\mathrm{H} 3$, and $5 \mathrm{nM}$ of either G9a or GLP as $30 \mu \mathrm{L}$ in black 96-well plate. After $5 \mathrm{~min}$, $20 \mu \mathrm{L}$ of SAM $(25 \mu \mathrm{M})$ in assay buffer was added. After incubation for $10 \mathrm{~min}$ at room temperature, thiol fluorescent probe IV $(20 \mu \mathrm{M}, 50 \mu \mathrm{L})$ was added. After $10 \mathrm{~min}$, the fluorescence of probe at Ex400/Em465 was measured by Infinite M Plex (Tecan, San Jose, CA, USA). Inhibition curves used for $\mathrm{IC}_{50}$ determination are reported in the Supplementary Materials (Figures S6 and S7).

\subsection{Computational Studies}

\subsubsection{Docking Studies}

The crystal structure of the Spindlin1 in complex with A366 (PDB ID: 6I8Y) was retrieved from the Protein Data Bank (PDB; www.rcsb.org (accessed on 17 March 2019)) [24] and prepared with Schrödinger's Protein Preparation Wizard tool [36]. Solvent molecules and sodium ions were removed; meanwhile, hydrogen atoms and missing side chain residues were added to the protein structure. Protonation states were assigned by PROPKA at $\mathrm{pH} 7.0$, and the hydrogen bonding network was consequently optimized. A restrained energy minimization step was then executed using the OPLS3 force field with default settings.

A Maestro 2D sketcher [37] was used to draw the ligand structures, which were then converted into 3D structures and prepared with Schrödinger's LigPrep tool [38]. All possible tautomeric forms and stereoisomers were generated at $\mathrm{pH} 7.0 \pm 1.0$ using Epik. Afterwards, a multi-conformational dataset was generated with ConfGen, allowing a maximum of 50 conformers per ligand and energy minimization of the output conformations using the default force field (OLPS_2005) [39,40].

Molecular docking studies were carried out by means of Glide using the standard precision (SP) mode [32]. Prior, a grid box was generated employing the Receptor Grid Generation tool, the co-crystallized A366 inhibitor was selected as the center of the grid, and a cube of $15 \AA$ was defined as the inner box. During docking, the option "sample ring conformation" was switched on, and a maximum of three docking poses were output for each ligand conformer; all other settings were kept as default. Moreover, the contribution 
of the intramolecular hydrogen bonds was evaluated and incorporated into the docking scores. This was done by allowing the option "reward intramolecular hydrogen bonds" in Glide [32]. In this way, the compounds that exhibit these intramolecular interactions pay a smaller entropic penalty upon the binding, which affects the final scores-referred to as DS_Intra-H-bonds in this article. Instead, DS refers to the default docking scores without the intramolecular hydrogen bonds' contribution. The above-mentioned protocol was able to reproduce the binding mode of $\mathbf{A} 36 \mathbf{6}$ as observed in the co-crystal structure with Spindlin1 with a root-mean-square deviation (RMSD, heavy atoms) of $0.54 \AA$ when using the top-scored pose (Supplementary Materials, Figure S1).

Box-plots were generated using the $\mathrm{R}$ package to evaluate the distribution of the docking scores (DS and DS_Intra-H-bonds) retrieved for the active and inactive ligands. Only the ligands with determined $\mathrm{IC}_{50}$ values were used as actives, whereas compounds 1c, $\mathbf{1 d}$, and $1 \mathrm{e}$ were treated as inactive.

\subsubsection{Molecular Dynamic Simulations}

The previously generated docking poses of A366, 1c, and 1e in complex with Spindlin1 (PDB ID: 6I8Y) were used as initial coordinates for the generation of the MD systems. The simulations were performed using the Amber18 package, and the systems were prepared by means of AmberTools18 tools [33]. First, topologies and force field parameters were assigned to the ligands with the Antechamber package using General Amber Force Field (GAFF) and AM1-BCC as atomic charges method (AM1-BBC, semi-empirical (AM1) with bond charge correction (BCC)) [41,42]. The ligands were considered in the positively charged state; thus, a net molecular charge of 2 was assigned to them. Then, each system was prepared in TLEaP using Amber ff03 force field for the protein and GAFF for the ligands. The systems were solvated in a TIP3P octahedral water box of $10 \AA$ and neutralized using $\mathrm{Na}^{+}$and counterions. The prepared systems were then subjected to a protocol that encompassed diverse steps. Initially, two consecutive minimization steps using constant-volume periodic boundaries were carried out. In each step, 4000 iterations (first 2000 steepest descent and then 2000 conjugate gradient) were applied to the system. In the first minimization step, only solvent atoms were minimized, while the protein and ligand atoms were restrained to their initial coordinates with a force constant of $10 \mathrm{kcal} \mathrm{mol}^{-1} \AA^{-2}$. In the second minimization step, the positional restraints were removed, and the whole system was minimized. Next, the system was heated, starting from a temperature of $0 \mathrm{~K}$ up to $300 \mathrm{~K}$ through $100 \mathrm{ps}$ of MD, applying constant-volume periodic boundaries. To prevent large structural deviations, the complex atoms (protein and ligand) were restrained with a force constant of $10 \mathrm{kcal} \mathrm{mol}^{-1} \AA^{-2}$. SHAKE was turned on and used to constrain bonds involving hydrogen [43]. The Langevin dynamics was employed to control the temperature using a collision frequency of $1 \mathrm{ps}^{-1}$. Then, after a density step, the system was subjected to an equilibration run of $2 \mathrm{~ns}$ with a time step of $2 \mathrm{fs}$ per step. The temperature and pressure were kept constant: $300 \mathrm{~K}$ and a constant-pressure periodic boundary maintained at an average pressure of $1 \mathrm{~atm}$ by using isotropic position scaling with a relaxation time of $2 \mathrm{ps}$. As for the heating step, SHAKE was turned, while the Langevin dynamics was employed to control the temperature using a collision frequency of $2 \mathrm{ps}^{-1}$.

Finally, each system was subjected to a production run of $200 \mathrm{~ns}$ under the same conditions used in the equilibration step (time step of $2 \mathrm{fs}$ per step, constant temperature of $300 \mathrm{~K}$, Langevin dynamics with a collision frequency of $2 \mathrm{ps}^{-1}$, constant-pressure periodic boundary with an average pressure of $1 \mathrm{~atm}$ using isotropic position scaling with a relaxation time of 2 ps). In all steps, a cutoff of $10 \AA$ was used for non-bonded interactions, and long-range electrostatic interactions were treated by means of the Particle Mesh Ewald (PME) method [44,45].

The trajectories were analyzed using the CPPTRAJ module [46] from AmberTools18 [33] and visualized in VMD. Plots were generated by means of $\mathrm{R}$ package. For the analysis of the protein-ligand hydrogen bonds, the default settings were used, which encompass a distance cutoff of $3 \AA$ A between the donor and acceptor atoms and an angle cutoff of 
135 degrees. Meanwhile, the distance cutoff was extended to $3.5 \AA$ for the analysis of the ligand intramolecular hydrogen bonds. Two criteria were applied to detect the presence of cation-pi interactions between the ligand and the residues of the aromatic cage (Phe141, Trp151, Tyr170, and Tyr177): (i) a distance cutoff of $6 \AA$ between the center of mass of the positively charged pyrrolidine nitrogen and the centroid of the aromatic ring and (ii) an angle cutoff of 45 degrees; the angle was defined as the angle between the normal vector of the aromatic ring plane and the vector connecting the positively charged pyrrolidine nitrogen and the centroid of the aromatic ring. Salt bridge interactions were considered to be formed if the distance between the negatively charged oxygen atom of Asp184 (or Asp189) and the positively charged amidine nitrogen of the ligand were within the cutoff distance of $4 \AA$.

All 100,000 frames retrieved from the $200 \mathrm{~ns}$ MDs were subjected to the analysis. The occupancy rates of the interactions were calculated as the percent of the frames in which a specific interaction was observed.

\subsubsection{Prime MM-GBSA}

The first docking poses were rescored with Prime MM-GBSA, a tool implemented in Schrödinger suite that performs protein-ligand complex minimization and refinement [47]. The VSGB solvation model and OPLS3 force field were used [48]. Several settings were tested, and we report to two different protocols: (i) only the ligands were relaxed, and (ii) also the protein residues within $3 \AA$ from the ligands were treated as flexible. For the analysis of the results, for the docking studies, box-plots were generated using the $R$ package to show the distribution of the computed binding affinity values (MM-GBSA $\Delta \mathrm{G}_{\text {bind }}$ ) obtained for the active and inactive ligands. Only the ligands with determined $\mathrm{IC}_{50}$ values were used as actives, whereas compounds $\mathbf{1 c}, \mathbf{1 d}$, and $1 \mathbf{e}$ were treated as inactive compounds.

\section{Conclusions}

In previous work, we reported the discovery of A366, an originally developed G9a inhibitor [30], as a nanomolar Spindlin1 inhibitor (Figure 1) [31]. Later, the crystal structure of Spindlin1 in complex with A366 was resolved (PDB ID: 6I8Y, [27]), and some modifications, as well as bivalent derivatives, were investigated [27]. In this study, we synthesized, tested, and computationally analyzed a series of 21 A366 derivatives as Spindlin1 inhibitors to further explore the SAR of this series. The main modification that had a drastic impact on the activity was the length of the linker chain, where the actives bear a propyl chain, while the inactive compounds either an ethyl or a butyl chain. Since the pyrrolidine function mimics the trimethylated lysine moiety, it is crucial for the inhibitory activity. Minor modification of the pyrrolidine ring did not lead to significant changes in the inhibitory activity (e.g., 1q), whereas its replacement with an isoindoline moiety yielded 1s, a good Spindlin1 binder, and the most selective compound of our series (Spindlin1 $\mathrm{IC}_{50}: 360 \pm 20 \mathrm{nM}, \mathrm{G} 9 \mathrm{a} \mathrm{IC} 50>20 \mu \mathrm{M}$, and GLP IC $50>20 \mu \mathrm{M}$ ). Likewise, a good selectivity profile was observed for compound $\mathbf{1 t}$ bearing a 3-methylpyrrolidine moiety and a 3-ethyl-3-methyl substitution on R4. Substituents on R2 position were not well tolerated, as highlighted by a significant decrease in the inhibitory activity of several compounds $(\mathbf{1 a}, \mathbf{1 h}, \mathbf{1} \mathbf{i}, \mathbf{1} \mathbf{j}$, and $\mathbf{1 k})$. Meanwhile, the replacement of the methoxy group at R1 displayed minor $(\mathbf{1} \mathbf{g}, \mathbf{1 1})$ to more significant $(\mathbf{1 m})$ decreases in the activity based on the substitutions. Finally, modifications of the spiro moiety showed no major impact on the inhibitory activity of the compounds; more relevant effects were observed with the geminal dimethyl and diethyl modifications. Notably, compound $1 \mathbf{p}$ was the most active derivative of the series (Spindlin1 $\mathrm{IC}_{50}: 157 \pm 12 \mathrm{nM}$ ) but showed no selectivity over G9a; while compound 1o exhibited a decreased activity for Spindlin1 coupled with an increase in selectivity over G9a and GLP (Spindlin1 IC 50 : $1.21 \pm 0.09 \mu \mathrm{M}$, G9a IC $50>20 \mu \mathrm{M}$, GLP IC $50>20 \mu \mathrm{M}$ ). The synthesis and in vitro testing were coupled with various computational approaches. Docking studies and MD simulations were carried out to rationalize the lack of activity 
of some derivatives. Interestingly, while from the predicted binding modes and docking scores it was not possible to explain the differences in the activity, the analysis of the MD simulations shed light on the important interactions and their contributions. Moreover, rescoring the docking poses with MM-GBSA calculations was successful in discriminating active from inactive compounds within our congeneric series of A366 inhibitors.

Considering the SAR of our series and the structural features of the binding site of Spindlin1 and G9a, future work will concentrate on attempting more substitutions on $\mathrm{R} 2$ and exploring other replacement for the pyrrolidine moiety. Specifically, longer and hydrophilic substitutions will be tested on R2, which are postulated, on the one hand, to be able to interact with the surrounding Spindlin1 residues (e.g., Asp95 and Glu142), and on the other clash with the smaller G9a pocket and ensure selectivity. Additionally, bisubstitutions on the pyrrolidine moiety as well as other bicyclic basic moieties will be probed to achieve further potent and selective compounds.

It was reported that high levels of Spindlin1 have been observed in liposarcoma and other types of tumors including ovarian cancer [13]. Thus, targeting the Spindlin1/ H3K4me3 pocket with small molecule inhibitors as described in the present work might be an interesting therapeutic approach for such cancer treatment. The developed potent and selective Spindlin1 inhibitors are drug-like molecules that can be used for further cellular studies to achieve this goal.

Supplementary Materials: The following are available online at https:/ / www.mdpi.com/article/10 $.3390 /$ ijms22115910/s1.

Author Contributions: C.L. and D.R. carried out the docking, molecular dynamics simulations and binding free energy calculations and wrote the manuscript. P.R., D.M. and D.O. synthesized compounds and provided them for in vitro testing. L.S., J.S., J.B., T.B. and T.W. developed and applied the Spindlin1 in vitro assays. H.G. and R.S. expressed and provided the proteins for testing. K.-S.P. and Y.X. carried out the G9a testing. B.B. supervised the synthesis of the compounds and the analytics. J.J. and M.J. supervised the in vitro testing and wrote the manuscript. W.S. designed and supervised the computational studies and was in charge of revising and reviewing the manuscript. All authors have read and agreed to the published version of the manuscript.

Funding: B.B., M.J. and R.S. received funding from the Deutsche Forschungsgemeinschaft (German Research Foundation, DFG Project-ID 192904750—SFB 992) and M.J. and R.S. under Germany's Excellence Strategy-EXC-2189-Project ID 390939984). R.S. was further supported by grants from the European Research Council (AdGrant 322844) and the DFG (CRC 850, CRC 746, and SCHU688/12-1).

Institutional Review Board Statement: Not applicable.

Informed Consent Statement: Not applicable.

Data Availability Statement: Not applicable.

Acknowledgments: We acknowledge the financial support of the Open Access Publication Fund of the Martin-Luther-University Halle-Wittenberg.

Conflicts of Interest: The authors declare no conflict of interest.

\section{References}

1. Wang, W.; Chen, Z.; Mao, Z.; Zhang, H.; Ding, X.; Chen, S.; Zhang, X.; Xu, R.; Zhu, B. Nucleolar protein spindlin1 recognizes H3K4 methylation and stimulates the expression of rRNA genes. EMBO Rep. 2011, 12, 1160-1166. [CrossRef] [PubMed]

2. Yang, N.; Wang, W.; Wang, Y.; Wang, M.; Zhao, Q.; Rao, Z.; Zhu, B.; Xu, R.-M. Distinct mode of methylated lysine-4 of histone H3 recognition by tandem tudor-like domains of spindlin1. Proc. Natl. Acad. Sci. USA 2012, 109, 17954-17959. [CrossRef] [PubMed]

3. Su, X.; Zhu, G.; Ding, X.; Lee, S.Y.; Dou, Y.; Zhu, B.; Wu, W.; Li, H. Molecular basis underlying histone H3 lysine-arginine methylation pattern readout by Spin/Ssty repeats of spindlin1. Genes Dev. 2014, 28, 622-636. [CrossRef]

4. Shanle, E.K.; Shinsky, S.A.; Bridgers, J.; Bae, N.; Sagum, C.; Krajewski, K.; Rothbart, S.B.; Bedford, M.T.; Strahl, B.D. Histone peptide microarray screen of chromo and Tudor domains defines new histone lysine methylation interactions. Epigenetics Chromatin 2017, 10, 12. [CrossRef] [PubMed]

5. Wang, C.; Zhan, L.; Wu, M.; Ma, R.; Yao, J.; Xiong, Y.; Pan, Y.; Guan, S.; Zhang, X.; Zang, J. Spindlin-1 recognizes methylations of K20 and R23 of histone H4 tail. FEBS Lett. 2018, 592, 4098-4110. [CrossRef] 
6. Zhao, F.; Liu, Y.; Su, X.; Lee, J.-E.; Song, Y.; Wang, D.; Ge, K.; Gao, J.; Zhang, M.Q.; Li, H. Molecular basis for histone H3 "K4me3-K9me3/2" methylation pattern readout by spindlin1. J. Biol. Chem. 2020, 295, 16877-16887. [CrossRef] [PubMed]

7. Barski, A.; Cuddapah, S.; Cui, K.; Roh, T.-Y.; Schones, D.E.; Wang, Z.; Wei, G.; Chepelev, I.; Zhao, K. High-resolution profiling of histone methylations in the human genome. Cell 2007, 129, 823-837. [CrossRef]

8. Wang, Z.; Zang, C.; Rosenfeld, A.J.; Schones, E.D.; Barski, A.; Cuddapah, S.; Cui, K.; Roh, T.-Y.; Peng, W.; Zhang, M.Q.; et al. Combinatorial patterns of histone acetylations and methylations in the human genome. Nat. Genet. 2008, 40, 897-903. [CrossRef]

9. Jiang, F.; Zhao, Q.; Qin, L.; Pang, H.; Pei, X.; Rao, Z. Expression, purification, crystallization and preliminary X-Ray analysis of human spindlin1, an ovarian cancer-related protein. Protein Pept. Lett. 2006, 13, 203-205. [CrossRef] [PubMed]

10. Chen, X.; Wang, Y.-W.; Xing, A.-Y.; Xiang, S.; Shi, D.-B.; Liu, L.; Li, Y.-X.; Gao, P. Suppression of SPIN1-mediated PI3K-Akt pathway by miR-489 increases chemosensitivity in breast cancer. J. Pathol. 2016, 239, 459-472. [CrossRef] [PubMed]

11. Drago-Ferrante, R.; Pentimalli, F.; Carlisi, D.; De Blasio, A.; Saliba, C.; Baldacchino, S.; Degaetano, J.; Debono, J.; Caruana-Dingli, G.; Grech, G.; et al. Suppressive role exerted by microRNA-29b-1-5p in triple negative breast cancer through SPIN1 regulation. Oncotarget 2017, 8, 28939-28958. [CrossRef]

12. Song, Q.; Ji, Q.; Xiao, J.; Li, F.; Wang, L.; Chen, Y.; Xu, Y.; Jiao, S. miR-409 inhibits human non-small-cell lung cancer progression by directly targeting SPIN1. Mol. Ther. Nucleic Acids 2018, 13, 154-163. [CrossRef]

13. Franz, H.; Greschik, H.; Willmann, D.; Ozretić, L.; Jilg, C.A.; Wardelmann, E.; Jung, M.; Buettner, R.; Schule, R. The histone code reader SPIN1 controls RET signaling in liposarcoma. Oncotarget 2015, 6, 4773-4789. [CrossRef] [PubMed]

14. Zhao, M.; Bu, Y.; Feng, J.; Zhang, H.; Chen, Y.; Yang, G.; Liu, Z.; Yuan, H.; Yuan, Y.; Liu, L.; et al. SPIN1 triggers abnormal lipid metabolism and enhances tumor growth in liver cancer. Cancer Lett. 2020, 470, 54-63. [CrossRef] [PubMed]

15. Chen, X.; Wang, Y.-W.; Gao, P. SPIN1, negatively regulated by miR-148/152, enhances Adriamycin resistance via upregulating drug metabolizing enzymes and transporter in breast cancer. J. Exp. Clin. Cancer Res. 2018, 37, 100. [CrossRef] [PubMed]

16. Gao, Y.; Yue, W.; Zhang, P.; Li, L.; Xie, X.; Yuan, H.; Chen, L.; Liu, D.; Yan, F.; Pei, X. spindlin1, a novel nuclear protein with a role in the transformation of NIH3T3 cells. Biochem. Biophys. Res. Commun. 2005, 335, 343-350. [CrossRef] [PubMed]

17. Zhang, P.; Cong, B.; Yuan, H.; Chen, L.; Lv, Y.; Bai, C.; Nan, X.; Shi, S.; Yue, W.; Pei, X. Overexpression of spindlin1 induces metaphase arrest and chromosomal instability. J. Cell. Physiol. 2008, 217, 400-408. [CrossRef] [PubMed]

18. Yuan, H.; Zhang, P.; Qin, L.; Chen, L.; Shi, S.; Lu, Y.; Yan, F.; Bai, C.; Nan, X.; Liu, D.; et al. Overexpression of SPINDLIN1 induces cellular senescence, multinucleation and apoptosis. Gene 2008, 410, 67-74. [CrossRef] [PubMed]

19. Fang, Z.; Cao, B.; Liao, J.-M.; Deng, J.; Plummer, K.D.; Liao, P.; Liu, T.; Zhang, W.; Zhang, K.; Li, L.; et al. SPIN1 promotes tumorigenesis by blocking the uL18 (universal large ribosomal subunit protein 18)-MDM2-p53 pathway in human cancer. eLife 2018, 7, e31275. [CrossRef] [PubMed]

20. Janecki, D.M.; Sajek, M.; Smialek, M.J.; Kotecki, M.; Ginter-Matuszewska, B.; Kuczynska, B.; Spik, A.; Kolanowski, T.J.; Kitazawa, R.; Kurpisz, M.; et al. SPIN1 is a proto-oncogene and SPIN3 is a tumor suppressor in human seminoma. Oncotarget 2018, 9, 32466-32477. [CrossRef]

21. Wang, J.-X.; Zeng, Q.; Chen, L.; Du, J.-C.; Yan, X.-L.; Yuan, H.-F.; Zhai, C.; Zhou, J.-N.; Jia, Y.-L.; Yue, W.; et al. SPINDLIN1 Promotes Cancer Cell Proliferation through Activation of WNT/TCF-4 Signaling. Mol. Cancer Res. 2012, 10, 326-335. [CrossRef]

22. Greschik, H.; Duteil, D.; Messaddeq, N.; Willmann, D.; Arrigoni, L.; Sum, M.; Jung, M.; Metzger, D.; Manke, T.; Günther, T.; et al. The histone code reader Spin1 controls skeletal muscle development. Cell Death Dis. 2017, 8, e3173. [CrossRef]

23. Choi, J.-W.; Zhou, W.; Nie, Z.-W.; Niu, Y.-J.; Shin, K.-T.; Cui, X.-S. Spindlin1 alters the metaphase to anaphase transition in meiosis I through regulation of BUB3 expression in porcine oocytes. J. Cell. Physiol. 2019, 234, 8963-8974. [CrossRef]

24. Berman, H.M.; Westbrook, J.; Feng, Z.; Gilliland, G.; Bhat, T.N.; Weissig, H.; Shindyalov, I.N.; Bourne, P.E. The protein data bank. Nucleic Acids Res. 2000, 28, 235-242. [CrossRef]

25. Zhao, Q.; Qin, L.; Jiang, F.; Wu, B.; Yue, W.; Xu, F.; Rong, Z.; Yuan, H.; Xie, X.; Gao, Y.; et al. Structure of human spindlin1. J. Biol. Chem. 2007, 282, 647-656. [CrossRef]

26. Bae, N.; Viviano, M.; Su, X.; Lv, J.; Cheng, D.; Sagum, C.; Castellano, S.; Bai, X.; Johnson, C.; Khalil, M.I.; et al. Developing spindlin1 small-molecule inhibitors by using protein microarrays. Nat. Chem. Biol. 2017, 13, 750-756. [CrossRef]

27. Fagan, V.; Johansson, C.; Gileadi, C.; Monteiro, O.; Dunford, J.E.; Nibhani, R.; Philpott, M.; Malzahn, J.; Wells, G.; Farham, R.; et al. A chemical probe for tudor domain protein spindlin1 to investigate chromatin function. J. Med. Chem. 2019, 62, 9008-9025. [CrossRef] [PubMed]

28. Xiong, Y.; Greschik, H.; Johansson, C.; Seifert, L.; Bacher, J.; Park, K.-S.; Babault, N.; Martini, M.; Fagan, V.; Li, F.; et al. Discovery of a potent and selective fragment-like inhibitor of methyllysine reader protein spindlin 1 (SPIN1). J. Med. Chem. 2019, 62, 8996-9007. [CrossRef]

29. Robaa, D.; Wagner, T.; Luise, C.; Carlino, L.; McMillan, J.; Flaig, R.; Schüle, R.; Jung, M.; Sippl, W. Identification and structureactivity relationship studies of small-molecule inhibitors of the methyllysine reader protein spindlin1. Chem. Med. Chem. 2016, 11, 2327-2338. [CrossRef] [PubMed]

30. Sweis, R.F.; Pliushchev, M.; Brown, P.J.; Guo, J.; Li, F.; Maag, D.; Petros, A.M.; Soni, N.B.; Tse, C.; Vedadi, M.; et al. Discovery and development of potent and selective inhibitors of histone methyltransferase G9a. ACS Med. Chem. Lett. 2014, 5, 205-209. [CrossRef] [PubMed] 
31. Wagner, T.; Greschik, H.; Burgahn, T.; Schmidtkunz, K.; Schott, A.-K.; McMillan, J.; Baranauskiene, L.; Xiong, Y.; Fedorov, O.; Jin, J.; et al. Identification of a small-molecule ligand of the epigenetic reader protein spindlin1 via a versatile screening platform. Nucleic Acids Res. 2016, 44, e88. [CrossRef] [PubMed]

32. Schrödinger Release 2017-1: Glide; Schrödinger, LLC: New York, NY, USA, 2017.

33. Case, D.A.; Ben-Shalom, I.Y.; Brozell, S.R.; Cerutti, D.S.; Cheatham, T.E., III; Cruzeiro, V.W.D.; Darden, T.A.; Duke, R.E.; Ghoreishi, D.; Gilson, M.K.; et al. AMBER 2018; University of California: San Francisco, CA, USA, 2018.

34. Xiong, Y.; Li, F.; Babault, N.; Wu, H.; Dong, A.; Zeng, H.; Chen, X.; Arrowsmith, C.; Brown, P.J.; Liu, J.; et al. Structure-activity relationship studies of G9a-like protein (GLP) inhibitors. Bioorganic Med. Chem. 2017, 25, 4414-4423. [CrossRef] [PubMed]

35. Collazo, E.; Couture, J.-F.; Bulfer, S.; Trievel, R.C. A coupled fluorescent assay for histone methyltransferases. Anal. Biochem. 2005, 342, 86-92. [CrossRef]

36. Schrödinger Release 2018-1: Protein Preparation Wizard; Epik, Schrödinger, LLC: New York, NY, USA; Impact, Schrödinger, LLC: New York, NY, USA; Prime, Schrödinger, LLC: New York, NY, USA, 2018.

37. Schrödinger Release 2018-1: Maestro; Schrödinger, LLC: New York, NY, USA, 2018.

38. Schrödinger Release 2018-1: LigPrep; Schrödinger, LLC: New York, NY, USA, 2018.

39. Schrödinger Release 2018-1: ConfGen; Schrödinger, LLC: New York, NY, USA, 2018.

40. Watts, K.S.; Dalal, P.; Murphy, R.B.; Sherman, W.; Friesner, R.A.; Shelley, J.C. ConfGen: A conformational search method for efficient generation of bioactive conformers. J. Chem. Inf. Model. 2010, 50, 534-546. [CrossRef]

41. Jakalian, A.; Bush, B.L.; Jack, D.B.; Bayly, C.I. Fast, efficient generation of high-quality atomic charges. AM1-BCC model: I. Method. J. Comput. Chem. 2000, 21, 132-146. [CrossRef]

42. Jakalian, A.; Jack, D.B.; Bayly, C.I. Fast, efficient generation of high-quality atomic charges. AM1-BCC model: II. Parameterization and validation. J. Comput. Chem. 2002, 23, 1623-1641. [CrossRef] [PubMed]

43. Ryckaert, J.-P.; Ciccotti, G.; Berendsen, H.J.C. Numerical integration of the cartesian equations of motion of a system with constraints: Molecular dynamics of n-alkanes. J. Comput. Phys. 1977, 23, 327-341. [CrossRef]

44. Darden, T.; York, D.; Pedersen, L. Particle mesh Ewald: An $N \cdot \log (N)$ method for Ewald sums in large systems. J. Chem. Phys. 1993, 98, 10089-10092. [CrossRef]

45. Essmann, U.; Perera, L.; Berkowitz, M.L.; Darden, T.; Lee, H.; Pedersen, L.G. A smooth particle mesh Ewald method. J. Chem. Phys. 1995, 103, 8577-8593. [CrossRef]

46. Roe, D.R.; Cheatham, I.T.E. PTRAJ and CPPTRAJ: Software for processing and analysis of molecular dynamics trajectory data. J. Chem. Theory Comput. 2013, 9, 3084-3095. [CrossRef]

47. Schrödinger Release 2018-1: Prime; Schrödinger, LLC: New York, NY, USA, 2018.

48. Harder, E.; Damm, W.; Maple, J.R.; Wu, C.; Reboul, M.; Xiang, J.Y.; Wang, L.; Lupyan, D.; Dahlgren, M.K.; Knight, J.L.; et al. OPLS3: A force field providing broad coverage of drug-like small molecules and proteins. J. Chem. Theory Comput. 2016, 12, 281-296. [CrossRef] [PubMed] 